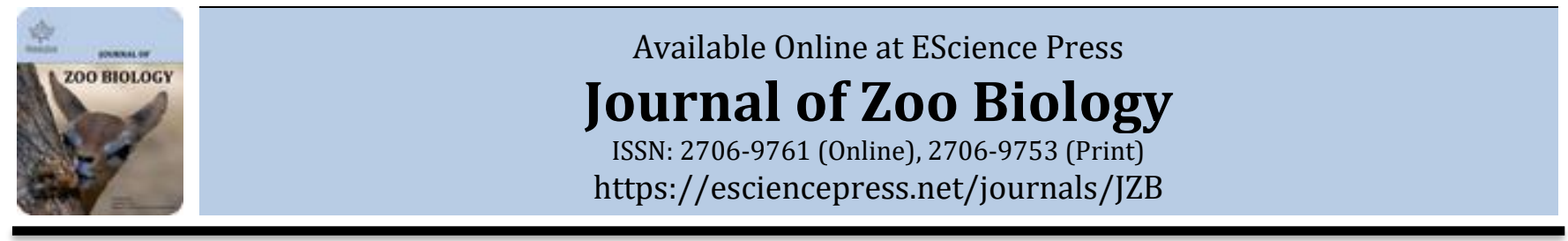

\title{
ENCLOSURE UTILIZATION AND ENRICHMENT STRUCTURE PREFERENCES OF CAPTIVE COYOTES
}

aJeffrey T. Schultz*, a,bulie K. Young

a USDA-National Wildlife Research Center-Predator Research Facility, Millville, Utah 84326 USA.

${ }^{b}$ Department of Wildland Resources, Utah State University, Logan, Utah, 84322-5295 USA.

*Corresponding Author Email: jeff.t.schultz@aphis.usda.gov Tel: +1-435-245-6091, x3112

\section{A B S T R A C T}

Environmental enrichment improves well-being of captive animals using a variety of tools, including adding complexity to the physical environment. Designing enrichment structures requires an understanding of behavioral and biological responses to enrichment efforts. Captive coyotes (Canis latrans) utilize shelter structures to hide, rest, and display vigilant behavior. Because these simple structures are regularly used, new and more complex enrichment structures could enhance enclosure enrichment. This study examined the time captive coyotes spent at discrete, complex enclosure features to determine: (1) how coyotes utilize enclosure space and shelter structures; and (2) if coyotes have a preferred enrichment structure design. Three enrichment structure designs (ramp, closed, and neutral) were installed simultaneously in 0.6-ha enclosures during two breeding seasons (January - March). Additional coyote pairs were monitored in control enclosures with simple structures. GPS-collars and scan sampling were used throughout a 28-day testing period to record space use and behavior. Coyotes spent most of their time at perimeter and open areas, but also exhibited a preference for shelter structures. Coyotes utilized the complex enrichment structures in treatment enclosures more than simple structures in control enclosures. Although there was no statistical preference for one specific type of complex structure, composite evidence from GPS-collars and behavioral data suggested that coyotes were most frequently located at ramp structures. Coyotes utilized ramp structures more during the daytime and demonstrated higher rates of vigilance there. This study advances the knowledge of captive coyote spatial patterns while helping improve environmental enrichment planning for captive facilities through the exploration of adding complexity to animal enclosures.

Keywords: Canis latrans, environmental enrichment, GPS collars, space use.

\section{INTRODUCTION}

The Association of Zoos and Aquariums (AZA, 2017) define enrichment as "a process to ensure that the behavioral and physical needs of an animal are being met by providing opportunities for species-appropriate behaviors and choices." This enhancement of an animal's environment likely improves the animal's psychological and physiological well-being, leading to increased welfare (Kuczaj et al., 2002). The implementation of environmental enrichment can improve an animal's ability to cope with acute stress and allow it to adapt to changing situations (Mellen and MacPhee, 2001). Environmental enrichment practices fall into several categories, including feeding regimes, toys, sensory stimulation, and physical environment
(Bloomsmith, Brent, and Schapiro, 1991; Newberry, 1995; Wells, 2009). Recording animal responses to enrichment efforts is often used to critically assess aspects of an enrichment program and can help judge the efficacy of enrichment efforts (Kuczaj et al., 2002; Mellen and MacPhee, 2001), although documentation procedures range from explicitly designed experiments to anecdotal annotations. Evaluation of these records can advance an enrichment program tailored to the preferences of the captive species, resulting in enhanced welfare and improved efficacy of husbandry efforts.

Captive animals inhabit fixed areas that can be categorized by function or environmental traits (i.e., edge, enrichment structure, feeding area). The proportion of space each 
category includes theoretically represents the expected proportions of the inhabitants' enclosure utilization if use matches availability of space. However, animals do not always match utilization of space to availability and instead show preferred (over-utilized) and avoided (underutilized) areas (Arjo and Pletscher, 2004; Beyer et al., 2010). When measured, over-utilized areas highlight environmental features that are desirable to the animal, while under-utilized areas reduce the effective size of an enclosure. This information is helpful for zoo managers who continuously seek to modify enclosure space to benefit animals in human care. Hunter, Gusset, Miller, and Somers (2014) found that captive African wild dogs (Lycaon pictus) preferred and avoided specific areas of their enclosures, depending on features such as substrate, slope, or proximity to zookeeper areas. Habitat utilization of California sea lions (Zalophus californianus) increased through olfactory enrichment that impacted the time they spent out of the water (Samuelson et al., 2016). Thus, the combination of environmental enrichment and effective enclosure design is best for animals in captivity (Law and Reid, 2010). Evaluating enclosure utilization can help assess the appropriateness of the environment in relation to biological and behavioral needs of captive animals (Ross, Schapiro, Hau, and Lukas, 2009). Since natural instincts may influence a captive animal's selection of resources, evaluating the utilization and functionality of enclosure areas and associated features can help managers improve the resources they provide and accommodate for speciesspecific inherent behavior.

Modification to an animal's physical environment to improve environmental enrichment efforts has been explored among several captive animal species, typically by providing additional structures to stimulate active wild behavior. General activity increased in spectacled bear (Tremarctos ornatus) by introducing climbing structures (Renner and Lussier, 2002) and in Indian leopards (Panthera pardus) with the provision of structurally enriched habitats compared to barren enclosures (Mallapur, Qureshi, and Chellam, 2002). Indian leopards housed in more complex enclosures also spent more time in the enriched zones of their enclosures compared to those in less complex enclosures (Mallapur et al., 2002). Similarly, red foxes (Vulpes vulpes) preferred areas having structural components over barren areas (Kistler, Hegglin, Würbel, and König, 2010). Increasing the complexity of enclosures also reduced the proportion of edge zone used by lion-tailed macaques (Macaca silenus) (Mallapur, Waran, and Sinha, 2005). Changes in enclosure utilization noted in these studies illustrate that enhanced areas that offer additional environmental choices are preferred by captive animals. A measured increase in the utilization of enriched areas is how captive animals express their preferences and infer a likely improvement of their welfare.

Understanding utilization of different enclosure features by captive animals can help managers gauge the biological relevance of unique environmental components and efficiently advance future designs of enclosures and enrichment structures. This study evaluated enclosure utilization of captive coyotes in light of the introduction of novel and more complex enrichment structures. Coyotes, especially during the breeding season, spend the largest proportion of their time inactive (Gese, Ruff, and Crabtree, 1996). They mainly rest during daytime hours and increase activity during nocturnal hours (Gese et al., 1996; Patterson, Bondrup-Nielsen, and Messier, 1999; Way, Ortega, and Strauss, 2004). Although coyotes are inactive for much of their winter daytime hours, it does not mean they sleep for this entire duration. Coyotes have been observed to often scan their surroundings while resting (Bekoff and Wells, 1981). Coyotes obey social hierarchies and are a highly territorial species (Gese and Ruff, 1997; Gilbert-Norton, Wilson, and Shivik, 2013). Thus, scanning while at rest may be used to watch territories for conspecific intruders, monitor pack mates, or search for prey. Several studies have evaluated wild coyote space use, home ranges, and habitat selection in relation to resources (Gese et al., 1996; Kluever and Gese, 2016; Mills and Knowlton, 1991; Shivik, Jaeger, and Barrett, 1996). Experiments observing captive coyotes have often measured behavior (Brummer, Gese, and Shivik, 2010; Gilbert-Norton, Leaver, and Shivik, 2009), and suggest similarities between captive and wild counterparts (Shivik, Palmer, Gese, and Osthaus, 2009). Research has yet to relate captive coyote behavior to enclosure feature utilization or measure their response to enrichment structures.

The United States Department of Agriculture (USDA)National Wildlife Research Center (NWRC)-Predator Research Facility in Millville, UT, USA, houses over 100 captive coyotes for research purposes. Simple structures, called shade tables, are provided within each outdoor enclosure. As wild coyotes would normally use structurally heterogeneous environmental features, captive coyotes at the research facility regularly utilize 
shade tables to hide, rest, and display vigilant behavior. Three novel enrichment structures were designed for this study to increase complexity within enclosures. To ensure the newly designed structures would be suitable for coyotes housed at the research facility, this study evaluated (1) how coyotes utilize enclosure space and shelter structures; and (2) if coyotes have a preferred enrichment structure design. Understanding how coyotes utilize resources and enclosure space will assist captive facilities with appropriately designing new enclosures and enrichment structures.

\section{MATERIALS AND METHODS}

Study overview: The study was conducted at the 164acre, USDA-NWRC Predator Research Facility in Millville, UT, USA, which houses over 100 adult coyotes in captivity as mated pairs for research purposes. Testing occurred during winter months (January - March) of 2015 and 2016. Thirty-two coyote pairs were randomly selected from all mated pairs in the captive colony, with 16 pairs tested each year. Males were vasectomized prior to the study, per facility standard operating procedures, to prevent successful breeding while housed with their mates for the experiment. Each pair of coyotes was randomly assigned to inhabit a treatment or control enclosure. Research protocols were approved by the Institutional Animal Care and Use Committee at the National Wildlife Research Center (QA-2375) and Utah State University (Protocol \#2490).

Eight 0.6 ha enclosures were utilized for this study for two 28-day periods in 2015 and 2016. The enclosures remained vacant for 1-3 days before experimental coyote pairs were released into the enclosures to allow for shelter structure construction and feces removal. Enclosures consisted of two human access gates and an animal capture kennel $(2 \mathrm{~m} \times 3 \mathrm{~m})$ with a concrete floor that was located at either the north or south corner (Figure 1). Each enclosure was comprised of natural substrate, an automatic watering device situated adjacent to one of the gates, and two den boxes made of cylindrical PVC $(0.5 \mathrm{~m}$ high $\times 0.5 \mathrm{~m}$ diameter $)$ providing corncob bedding (Green Products Company, Conrad, IA, USA) in each capture kennel. Only experimental or control shelter items were provided in the main enclosure area, and inground den holes were collapsed or otherwise made inaccessible during the study. Coyotes were scatter-fed normal daily rations ( $650 \mathrm{~g}$ per coyote) of a commercially prepared food (Fur Breeders Agricultural Cooperative,
Logan, UT, USA) in one specified area of each enclosure, and water was available ad libitum.

\section{Enrichment structures}

Control enrichment structures: Two study enclosures were randomly selected to serve as control enclosures. Control enclosures reflected shelter resources routinely available to captive coyotes by providing two wooden shade tables ( $0.6 \mathrm{~m}$ tall $\times 0.8 \mathrm{~m}$ wide $\times 1.2 \mathrm{~m}$ long) per enclosure. Shade table locations in the control enclosures were randomly assigned to two of the three locations designated for experimental shelter structures (Figure 1). Treatment enrichment structures: Enrichment structures were randomly assigned to occupy the three predetermined shelter locations in the treatment enclosures. The structures were simultaneously offered and spaced 40-55 m from each other and $>10 \mathrm{~m}$ from the perimeter fence (Figure 1). Middle points of the structure locations were recorded using a Garmin GPSMap 64® handheld device. All experimental enrichment structures included two components: (1) a wooden shade table and (2) an additional taller plywood platform (1.2 m x $1.2 \mathrm{~m})$ supported $1.2 \mathrm{~m}$ above the ground using four steel T-posts (Figure 2). Combining the two components, each enrichment structure spanned $4 \mathrm{~m}$ in length. Enrichment structures were oriented in a north-south direction, with the taller component positioned to the north. The three structure designs were: (1) a neutral structure composed of the basic two components, (2) a ramp structure that joined the two components using a $4 \mathrm{~cm} \times 24 \mathrm{~cm} \times 2.4 \mathrm{~m}$ wooden board, and (3) a closed structure formed by adding three plywood boards to the T-posts underneath the taller platform (Figure 2). Coyotes were allowed access into the closed cavity from the south and could access the top of the taller component with the ramp design.

\section{Data collection}

Global Positioning System (GPS) collars: Each coyote was fitted with a GPS-logger (i-gotU GT-600®, Mobile Action Technology, Inc.) for the 28-day test period. The logger was protected and attached via a vinyl pouch to a durable leather dog collar ( $3 \mathrm{~cm}$ wide), that was placed on the coyotes such that the device would face upward when the coyote was standing or lying in a prone position. Geographic coordinate locations for each coyote were recorded at 5-min intervals. Acquisition rates were also assessed for each coyote GPS-collar during each 28-day test period. 


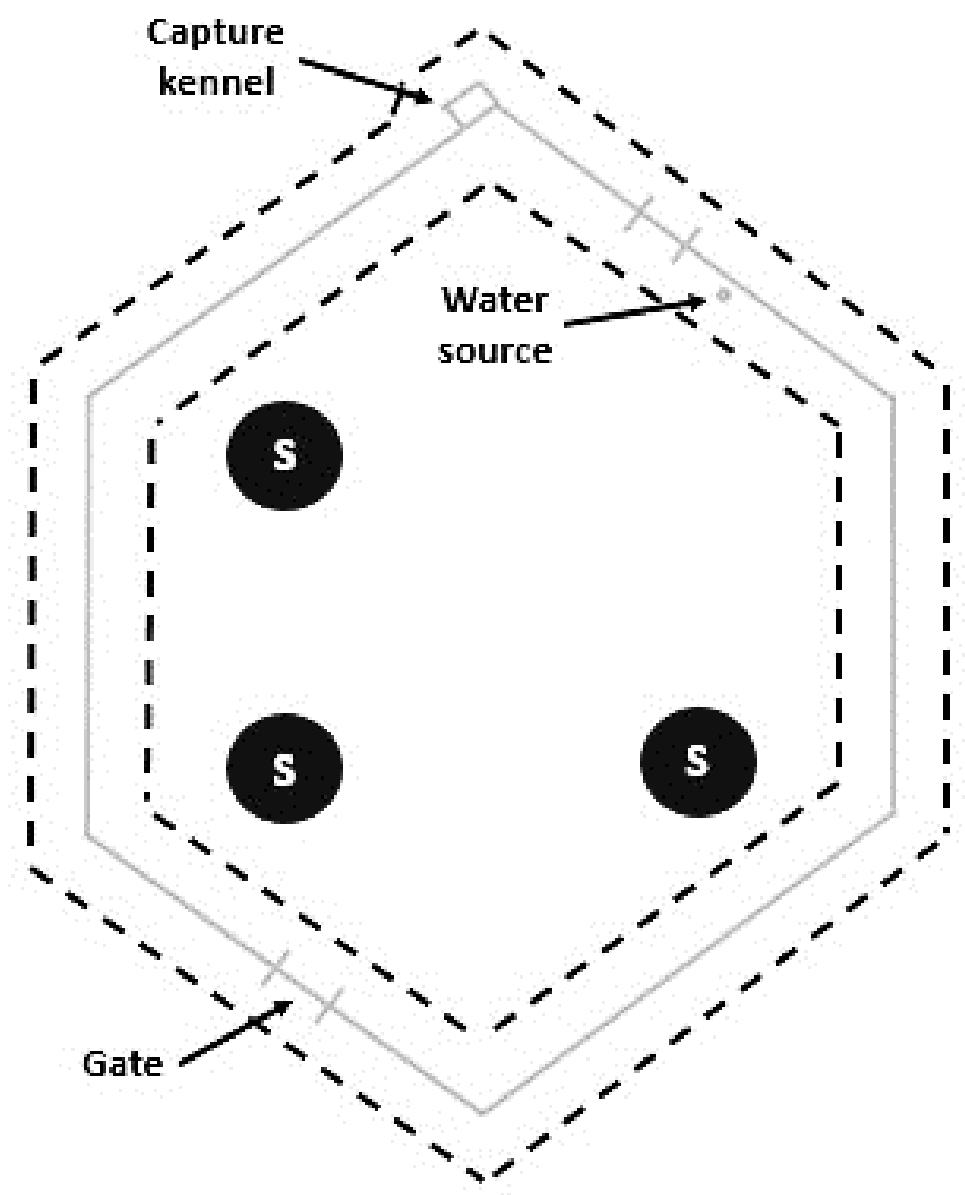

Figure 1. Enclosure used in January-March 2015 and 2016 for study on captive coyotes at the USDA-NWRC-Predator Research Facility in Millville, Utah. Locations of enrichment structures are depicted as S (dark circles denoting a 5-m buffer around the middle point of each shelter structure), perimeter as dashed lines (delineating a 5-m buffer on both sides of the enclosure fence to accommodate for GPS error), and open area as other interior space. Depiction is not to scale.

(a)
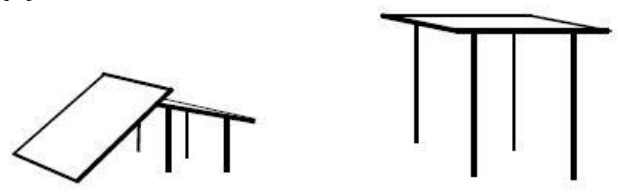

(b)

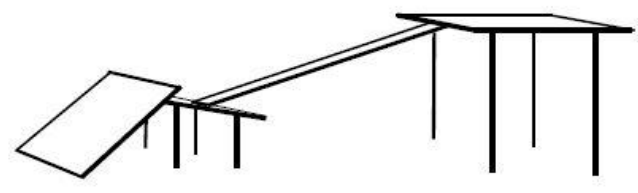

(c)

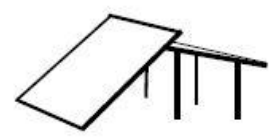

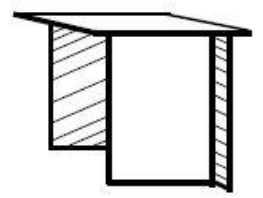

Figure 2. Sketches of three enrichment shelter structures provided to captive coyotes for testing: (a) neutral, (b) ramp, and (c) closed. Captive coyotes were previously exposed to shade tables, the shorter component of the enrichment structures, used in control enclosures. 
Nine GPS-collars were positioned at known geographic coordinates for accuracy testing during the second year of the study. To simulate potential positions of the GPSlogger attached to the coyotes, three collars were positioned so the GPS-logger was facing upward, three collars were set so the GPS-logger was facing parallel to the ground, and three collars were set facing the ground. Locations were recorded every five minutes for 28 days. The number of GPS points counted in a 5-m radius of the known geographic coordinate was divided by the total number of GPS points used. The resulting proportion represented the accuracy for the nine test collars, and a mean accuracy for each position was obtained by averaging the accuracies of the three collars that were set at the same position. Acquisition rates were also determined for the nine test collars and averages were obtained for the three test positions.

Behavioral observations: Scan sampling was used for all behavioral observations (Altmann, 1974) using a mobile observation blind that had been used for previous observational studies at the research facility. To avoid a response by coyotes to the blind, it was parked as far from the pens as possible while maintaining visibility of all enclosures using binoculars. Scans of each animal were conducted at 5-min intervals for one hour per day, four days per week, over the duration of each 28-day period. Although the coyotes appeared to ignore the observation blind, the observer arrived at the designated vantage point 15 min before beginning any observations to assure coyotes resumed their normal activities if they responded to the blind. Start times were randomly selected between 08:00 and 15:00 to ensure sufficient light for visibility. At each scan, the location and behavior of the study coyote was logged. Coyotes were recorded at enrichment structures when they were within $2 \mathrm{~m}$ of a structure and were considered at the perimeter when they were within 2 $\mathrm{m}$ of the perimeter fence. An ethogram was modified from Brummer et al. (2010), who monitored captive coyotes, and Gese et al. (1996), who observed wild coyotes. Behavior was then collapsed into three groups: vigilant, inactive, and active (Table 1). Only one person conducted all scans to eliminate inter-observer variability.

Table 1. Description of behavior categories used for analysis from scan observations.

\begin{tabular}{ll}
\hline \multicolumn{1}{c}{ Behavior category } & \multicolumn{1}{c}{ Description } \\
\hline Vigilant & $\begin{array}{l}\text { Lying, sitting, standing, walking, or running with head raised and visually surveying the } \\
\text { environment. } \\
\text { Lnactive }\end{array}$ \\
Lying and resting with head down or eyes closed (not vigilant); lying and grooming, sniffing \\
or biting grass; sitting; standing and drinking or grooming. \\
General & $\begin{array}{l}\text { Running; walking; pacing; digging; sniffing with nose close to the ground while walking or } \\
\text { standing. } \\
\text { Breeding activities (i.e., mounting, sniffing); dominant or subordinate playing or fighting; } \\
\text { howling. } \\
\text { Territorial }\end{array}$ \\
& $\begin{array}{l}\text { Marking (i.e., urinating or defecating then scratching, laying and rolling); stalking } \\
\text { conspecifics; tail flagging; fence running with vigilance directed at conspecifics. }\end{array}$ \\
\hline
\end{tabular}

Data analysis: GPS data were downloaded using @trip PC software (provided with the GPS-logger) and managed in ArcGIS®, version 2.2.2 (ESRI, 2014). Accuracy of the experimental collars was estimated by dividing the number of GPS points located within a $5 \mathrm{~m}$ buffer of a known geographic coordinate by the total number of points obtained from each collar. The first 12 hours of all GPS data used in this study was removed from analysis to allow time for the data loggers to initialize and find satellites.

Enclosure perimeters were delineated using editing tools in ArcGIS to trace the fence lines demarcated on the Environmental Systems Research Institute (ESRI) satellite basemap. Five-meter buffers were placed around the center points of each structure and along both sides of the enclosure perimeters to prevent overlapping (Figure 1). Coyote locations were categorized at discrete enclosure areas, including perimeter and enrichment structure, when coordinates from their GPS-collar fell within or intersected the buffer (Figure 1). All other locations inside the enclosure were categorized as open areas (Figure 1). Enclosure space comprised of 39\% perimeter space, $58 \%$ open areas, and $1 \%$ per structure. Thus, in control enclosures where there were only two structures, the open area made up 59\% of the enclosure. 
These proportions of available enclosure area space were derived using ArcGIS to represent the expected enclosure feature utilization for each individual. The observed proportion of time recorded at each enclosure feature for each coyote was obtained by dividing the number of GPS points at each feature by the total number of GPS points for each individual. For all GPS data, locations that fell outside of the perimeter buffer were excluded from analysis. Since the observed data did not follow normal distributions, non-parametric Mann-Whitney U-tests were performed to determine significant differences in observed proportions of GPS points at each enclosure feature between (1) treated animals $(n=60)$ and control animals ( $n=4)$, and (2) observed and expected enclosure feature utilization for treated and control animals.

A mixed logit model was fitted using the glmer function in the lme4 package, version 1.1-12 (Bates et al., 2015) in Program R, version 3.3.2 (R Core Team, 2016) to compare the probability of use among shelter structure locations between treatment and control enclosures. Using a binary response for structure use (yes/no) and the logit link function, fixed factors included sex (female/male), enclosure type (control/treatment), and time of day (day/night), and all interactions were included in the model. Day locations were from 600-1800 and night locations were from 1800-600. Random effects included individual and pair identifications to account for clustering within these groups. Predicted probabilities were obtained using the lsmeans function in the lsmeans package, version 2.5 (Lenth, 2016) in Program R.

To estimate coyote preferences among the three enrichment structure designs, a set of three generalized linear mixed models (GLMMs) fitted using binomial distributions and logit link functions were independently assembled to emulate the logistic equations that would simultaneously be estimated in a mixed multinomial regression model (Begg and Gray, 1984) using the GLIMMIX procedure in SAS/STAT®, version 14.2 (SAS Institute, 2013). Only GPS points falling within enrichment structure locations were used for the set of three GLMMs. Odds-ratio estimates were compiled to understand the utilization of one enrichment structure design in relation to another: (1) ramp use over neutral use, (2) ramp use over closed use, and (3) neutral use over closed use. The models included the same fixed factors as the logit model comparing shelter structure utilization between the control and treatment coyotes. To accommodate correlation due to clustering of GPS points within pairs, pair was included as a random effect. Using the GPS-collar data from points only at the enrichment structures, Mann-Whitney U-tests explored significant differences in observed proportions of GPS points between (1) males and females and (2) day and night structure utilization.

An additional GLMM using behavioral scans observed at the enrichment structures was fitted with a negative binomial distribution. There was no apparent difference in behavior between the male and female coyotes within a pair, so sex was not included as a predictor variable in the model. The response variable was scan count, summed over all observations for both coyotes in a pair. Behavior type (vigilant/inactive/active) and location (closed/neutral/ramp) were fixed effects factors, and the interactions between these factors were included in the model. Pair was again included as a random effect. The model was fitted using the glmmadmb function in the glmmADMB package, version 0.8.3.3 (Skaug, Fournier, Nielsen, Magnusson, and Bolker, 2013) in R. Means were estimated using the lsmeans function in the lsmeans package, and comparisons among means were computed using the contrast function in the lsmeans package. Family-wise Type I error was controlled using the Tukey method. The significance threshold was set at 0.05 for all statistical analysis.

\section{RESULTS}

GPS-collar accuracy: Mean proportions of GPS points found within a 5-m radius of a known point were 0.48 ( \pm $0.03)$ when the collar was facing the ground, $0.81( \pm 0.02)$ when it faced the sky, and $0.53( \pm 0.13)$ when the collar was facing parallel with the ground. Most GPS-collars recorded data at the programmed 5-minute intervals for the entire 28-day testing period. Acquisition rates of the GPS-collars on coyotes were $0.87( \pm 0.02)$, resulting in an average of $7356( \pm 150)$ locations per coyote. Of all acquired locations from GPS-collars on coyotes, an average of $0.83( \pm 0.01)$ of total GPS points fell within the enclosure area and were used for analysis. For test collars, acquisition rates were $0.96( \pm 0.003)$ for collars in the up position, $0.95( \pm 0.01)$ for collars in the side position, and $0.91( \pm 0.01)$ for collars that faced the ground.

Enclosure space use: Comparing coyotes in treatment enclosures to coyotes in control enclosures, treatment coyotes utilized the perimeter significantly less $(\mathrm{U}=50.0, \mathrm{P}$ $=0.05)$ and utilized structures significantly more $(U=4.0$, $\mathrm{P}<0.01$ ) (Figure 3). Comparing observed enclosure feature utilization to expected enclosure feature 
utilization, control coyotes significantly over-utilized shade tables $(\mathrm{U}=16, \mathrm{P}=0.01$ ) (Figure 4). Treatment coyotes significantly over-utilized enrichment structures $(\mathrm{U}=3480, \mathrm{P}<0.01)$ and significantly under-utilized open areas $(\mathrm{U}=1080, \mathrm{P}<0.01)$ (Figure 4).

Enrichment structure use: In treatment enclosures, the proportion of coyote locations at an enrichment structure was $0.12( \pm 0.00)$, while the proportion of coyote locations at a shade table in the control enclosures was $0.04( \pm 0.00)$ (Figure 3). Of accounts at enrichment structures, ramp structures had the highest proportion of use $(0.41 \pm 0.04)$, followed by neutral $(0.33 \pm 0.03)$ and closed structures $(0.27$ \pm 0.03 ). Experimental shelter structures were significantly over-utilized (ramp, $\mathrm{U}=2400, \mathrm{P}<0.01$; closed, $\mathrm{U}=2160$, $\mathrm{P}=$ 0.04; neutral, $\mathrm{U}=2400, \mathrm{P}<0.01$; shade table, $\mathrm{U}=16, \mathrm{P}=$ 0.01) (Figure 5). No significant differences in enrichment structure utilization were noticed from the proportions of GPS-collar locations between males and females (Figure 6). Coyotes utilized the ramp significantly more during the day $(\mathrm{U}=2229, \mathrm{P}=0.02)$ (Figure 7).

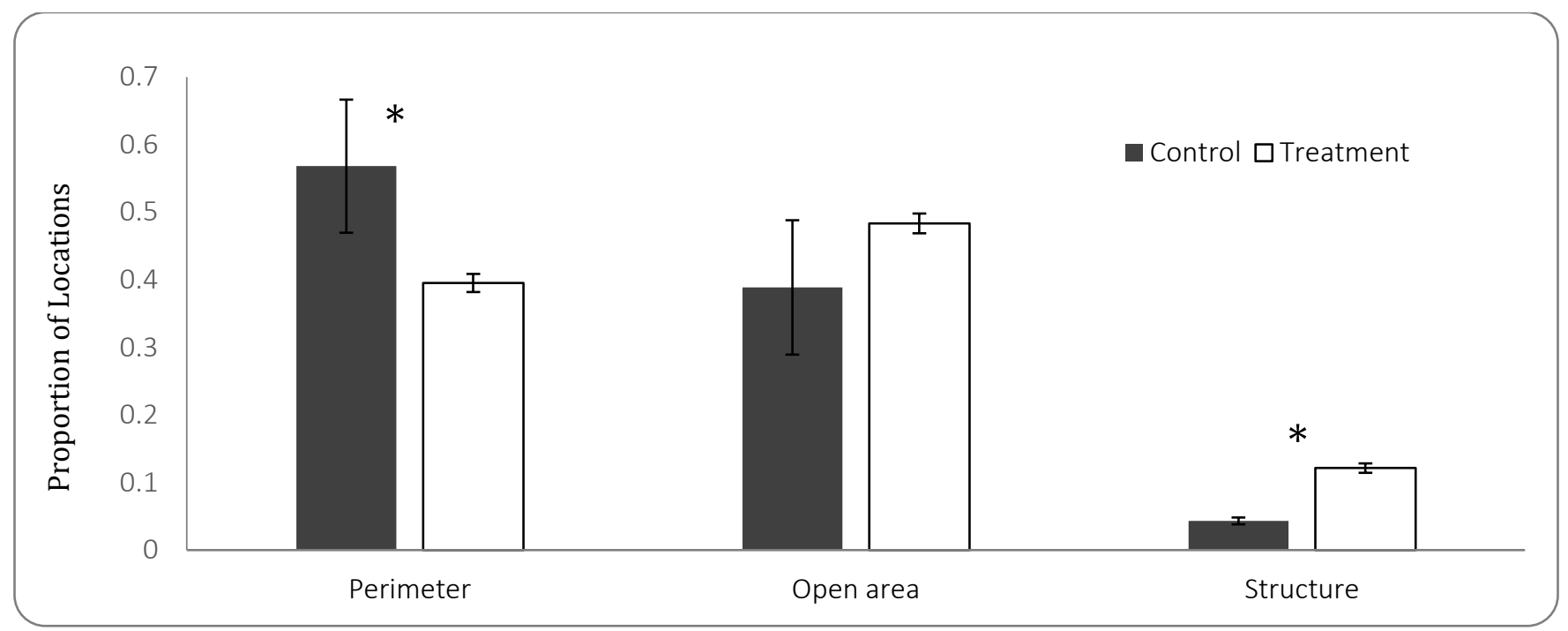

Figure 3. Average proportion of time spent at study enclosure features by mated pairs of captive coyotes. Three enrichment structures were installed in treatment enclosures while only two structures were in the control enclosures. Error bars represent standard error and $\left(^{*}\right)$ depict significant differences between control and treatment values.

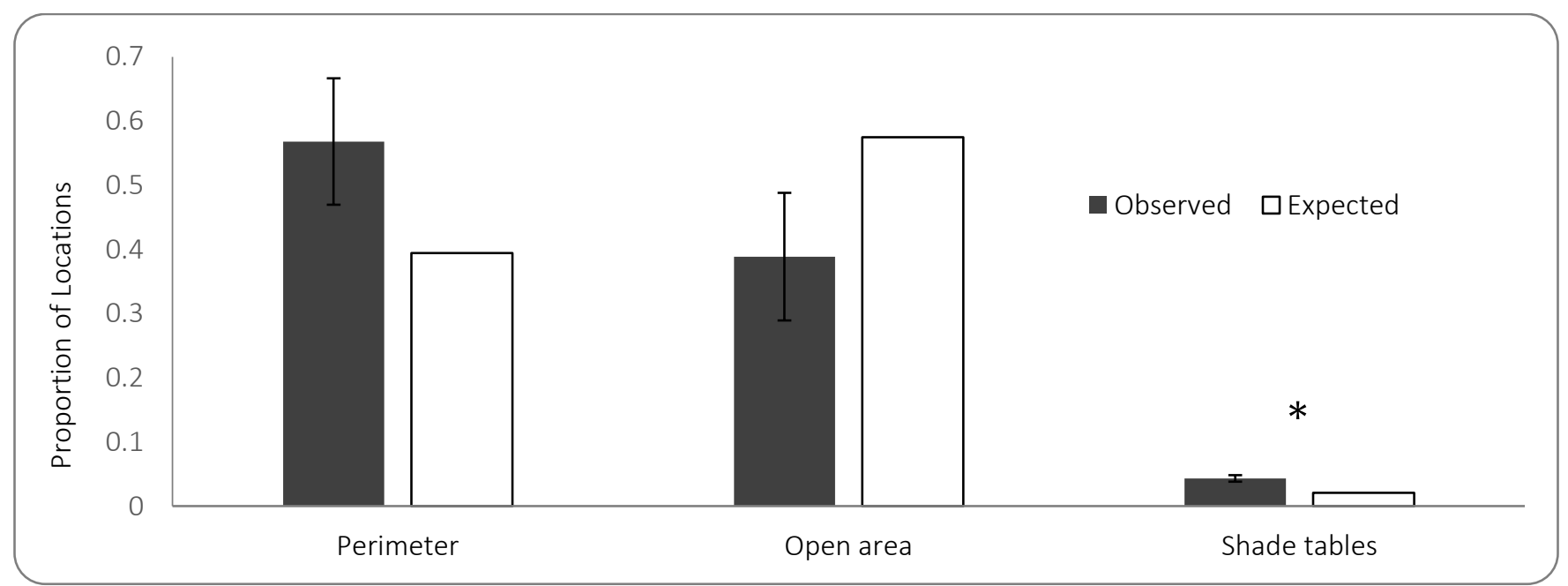

Figure 4(a). Average proportion of time spent at study enclosure features, compared to proportion of available space, for pairs of captive coyotes housed in control enclosures. Three enrichment structures were installed in treatment enclosures while only two structures were in the control enclosures. Error bars represent standard error and $\left({ }^{*}\right)$ depict significant differences between observed and expected values. 


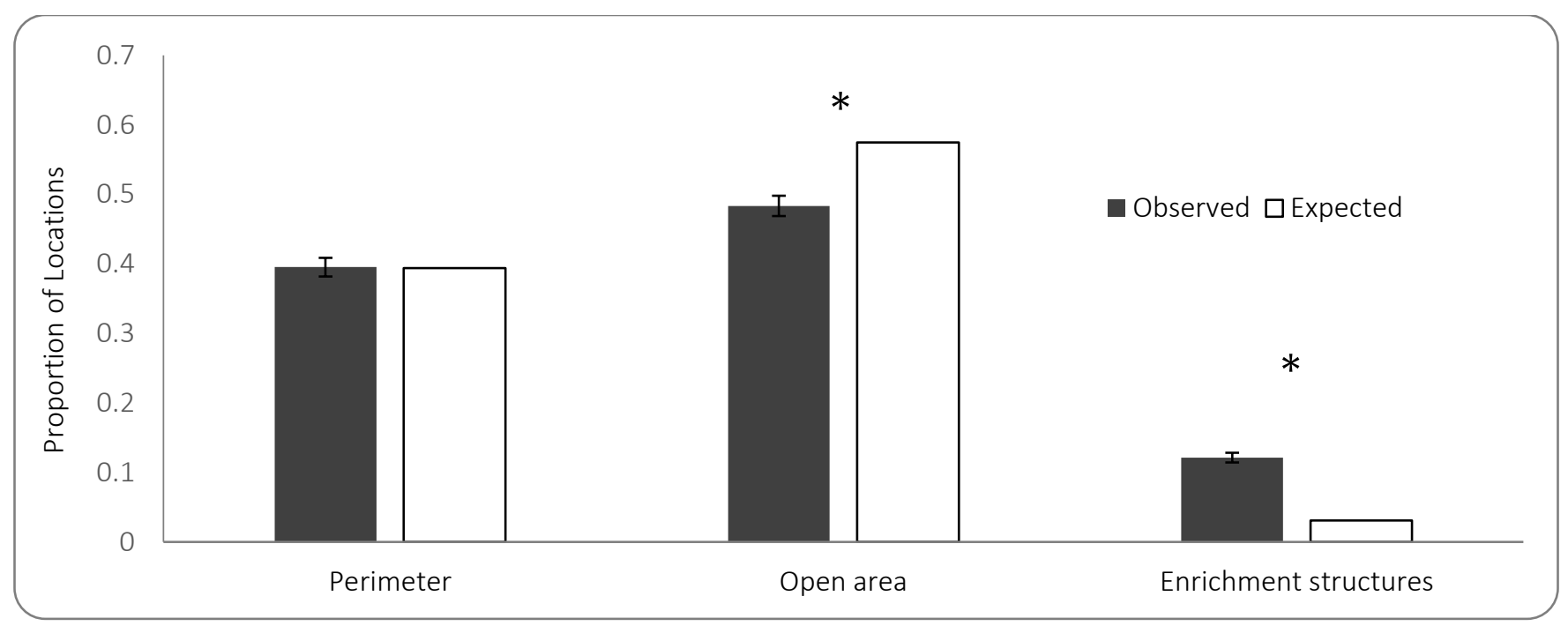

Figure 4(b). Average proportion of time spent at study enclosure features, compared to proportion of available space, for pairs of captive coyotes housed in treatment enclosures. Three enrichment structures were installed in treatment enclosures while only two structures were in the control enclosures. Error bars represent standard error and $(*)$ depict significant differences between observed and expected values.

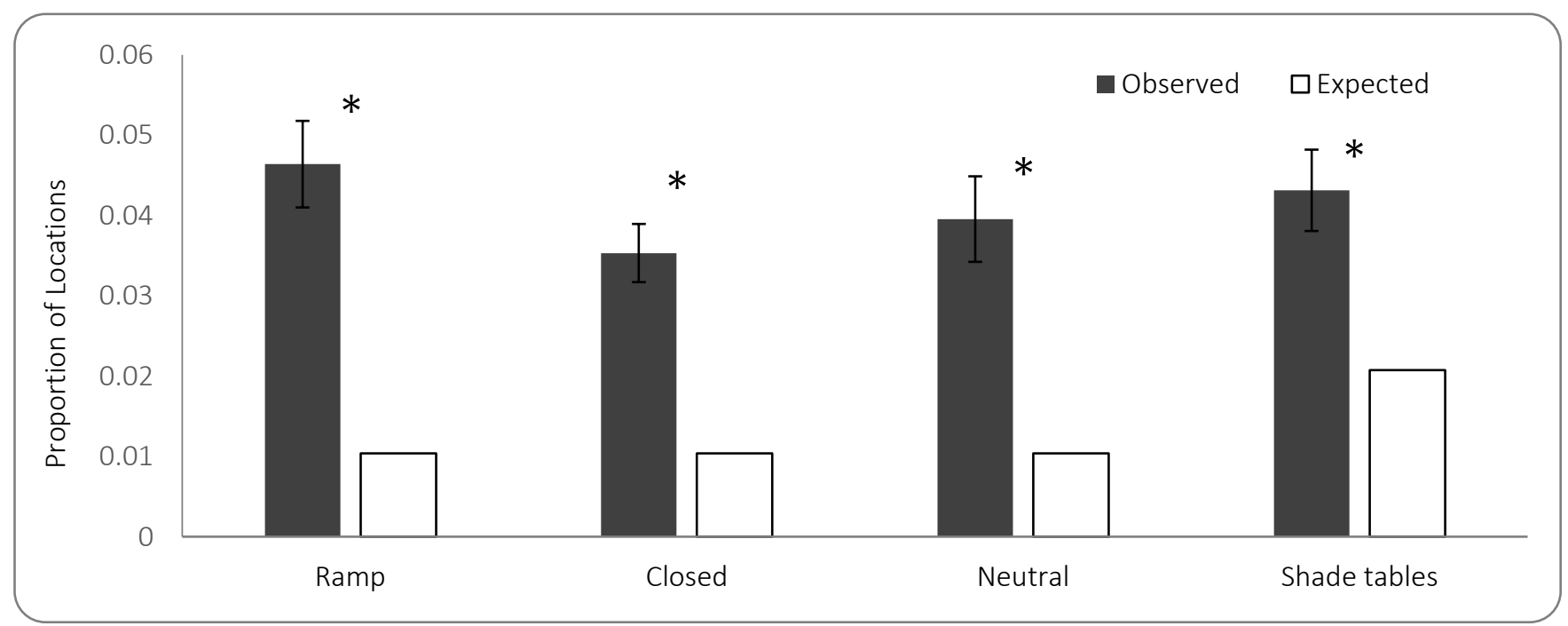

Figure 5. Average proportion of time mated pairs of captive coyotes spent at each type of shelter structure, compared to proportion of available space. One ramp, closed, and neutral structure was installed in each treatment enclosure while two shade tables were placed in each control enclosure. Error bars represent standard error and $\left({ }^{*}\right)$ depict significant differences between observed and expected values. 


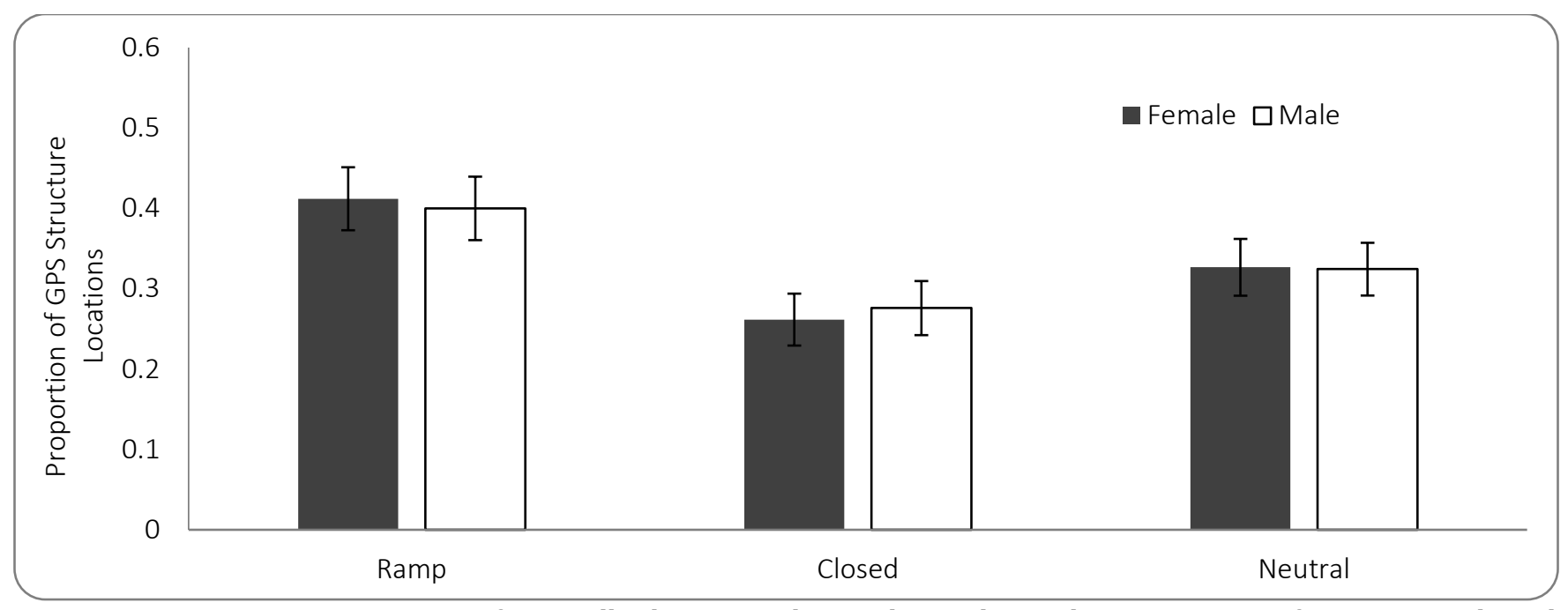

Figure 6. Mean average proportion of GPS-collar locations observed at each enrichment structure for captive male and female coyotes. Error bars represent standard error (SE) of individual mean proportions, and only GPS-collar locations at enrichment structures were used. Means and SEs shown are computed by descriptive statistics that used raw data and are not least squares means estimated by generalized linear mixed models.

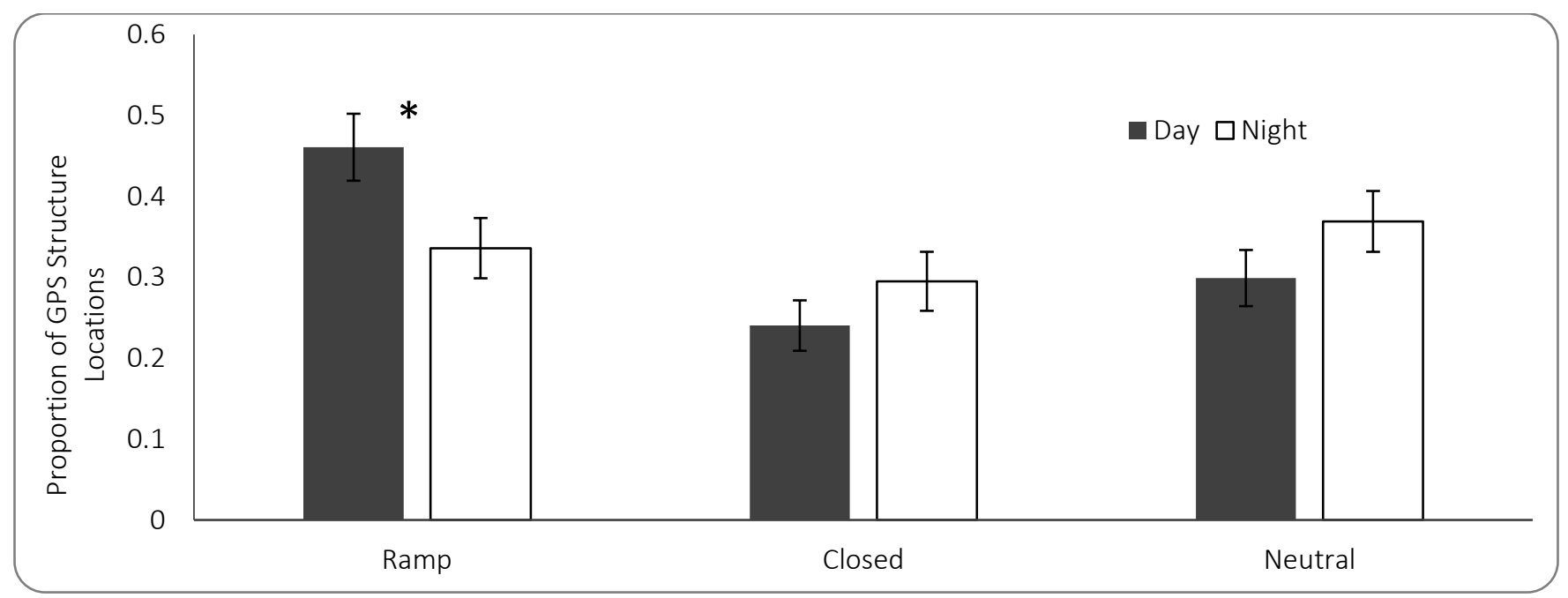

Figure 7. Mean average proportion of GPS-collar locations of captive coyotes at each enrichment structure by time of day. Error bars represent standard error (SE) of individual mean proportions, and ${ }^{*}$ ) depict significant differences between daytime and nighttime values. Only GPS-collar locations at enrichment structures were used. Means and SEs shown are computed by descriptive statistics that used raw data and are not least squares means estimated by generalized linear mixed models.

Results from the mixed logit model showed the treatment/control factor was a significant predictive term $(\mathrm{P}=0.01)$. The probability that control coyotes would be located at a shade table was 0.04 (95\% CI: 0.02 to 0.07 ) and the probability that treatment coyotes would be located at an enrichment structure was higher, estimated at 0.09 ( $95 \% \mathrm{CI}: 0.07$ to 0.10$)$.

Significant predictor variables varied among the three logistic regression models comparing the enrichment structure designs. Time of day $(P<0.01)$ and the interaction of time of day and sex $(\mathrm{P}<0.01)$ were significant in the model to explain ramp use compared to neutral use (Table 2). However, the models comparing ramp over closed structures and neutral over closed structures provided no statistical evidence that enrichment structure utilization varied by sex or time of day. The relative preference for ramp over neutral was higher during the day than at night, regardless of sex, but the relative preference was more pronounced for females (Table 3). 
Table 2. Tests of main effects and interactions of three generalized linear mixed models derived from GPS-collar data and used to predict odds ratios of relative enrichment structure utilization by captive coyotes. Only GPS-collar points at enrichment structures were used.

\begin{tabular}{lccr}
\hline Effect & df & $X^{2}$ & $P$ \\
\hline Neutral over Ramp: & & & 0.29 \\
\hline Sex & 1 & 1.18 & $<\mathbf{0 . 0 1}$ \\
Time of day & $\mathbf{1}$ & $\mathbf{1 3 . 6 8}$ & $<\mathbf{0 . 0 1}$ \\
Time of day * sex $^{\text {Closed } \text { over Ramp: }}$ & $\mathbf{1}$ & $\mathbf{2 1 . 6 2}$ & 0.19 \\
\hline Sex & & 1.84 & 0.06 \\
Time of day & 1 & 3.89 & 0.07 \\
Time of day ${ }^{\text {sex }}$ & 1 & 3.52 & 0.70 \\
\hline Closed over Ramp: & 1 & & 0.43 \\
Sex & & 0.15 & 0.97 \\
Time of day & 1 & 0.64 & \\
Time of day * sex & 1 & 0.00 & \\
\hline
\end{tabular}

${ }^{a}$ Bold denotes significance at the 0.05 level.

Table 3. Odds ratio of enrichment structure utilization, lower and upper bounds for a $95 \%$ confidence interval for the odds ratio, and p-value for the test of whether the odds ratio is different than one. Bold denotes significance at 0.05 .

\begin{tabular}{lcccc}
\hline Effect & Odds ratio & Lower bound & Upper bound & $P$ \\
\hline Ramp use over neutral use: & & & & 0.29 \\
\hline Female over Male & 1.12 & 0.91 & $\mathbf{1 . 4 3}$ & $\mathbf{0 . 0 0}$ \\
Day over night & $\mathbf{2 . 2 1}$ & $\mathbf{1 . 4 3}$ & $\mathbf{1 . 0 7}$ & $\mathbf{0 . 0 1}$ \\
Day, Female over Male & $\mathbf{1 . 3 3}$ & 0.75 & 1.17 & 0.55 \\
Night, Female over Male & 0.94 & $\mathbf{1 . 6 8}$ & $\mathbf{4 . 1 0}$ & $\mathbf{0 . 0 1}$ \\
Female, Day over Night & $\mathbf{2 . 6 3}$ & $\mathbf{1 . 1 9}$ & $\mathbf{2 . 9 0}$ & $\mathbf{0 . 0 1}$ \\
Male, Day over Night & $\mathbf{1 . 8 6}$ & & & 0.19 \\
\hline Ramp use over closed use: & & 0.93 & 3.46 & 0.06 \\
\hline Female over Male & 1.16 & 0.98 & 1.61 & 0.06 \\
Day over night & 1.73 & 0.99 & 1.37 & 0.58 \\
Day, Female over Male & 1.26 & 0.84 & 3.35 & 0.03 \\
Night, Female over Male & 1.07 & 1.06 & 2.84 & 0.11 \\
Female, Day over Night & 1.88 & 0.90 & & 0.70 \\
Male, Day over Night & 1.59 & & 1.48 & 0.43 \\
Neutral use over closed use: & & 0.76 & 1.50 & 0.71 \\
\hline Female over Male & 1.06 & 0.45 & 1.49 & 0.72 \\
Day over night & 0.80 & 0.76 & 1.43 & 0.44 \\
Day, Female over Male & 1.07 & 0.76 & 1.43 & 0.43 \\
Night, Female over Male & 1.06 & 0.45 & & \\
Female, Day over Night & 0.80 & 0.45 & & \\
Male, Day over Night & 0.80 & & & \\
\hline
\end{tabular}

Results from the GLMM derived from behavioral observations showed significant differences in the distribution of coyote enrichment structure selection and behavior $(\mathrm{P}<0.01)$, along with the distribution of behavior at the varying enrichment structures $(\mathrm{P}=0.01)$. Pairwise comparisons show coyotes selected the ramp significantly 
more often than the closed $(\mathrm{P}<0.01)$ and neutral structures $(P<0.01)$. Inactive behavior was significantly more frequent than vigilant behavior $(\mathrm{P}<0.01)$, and vigilant behavior was significantly more frequent than active behavior $(\mathrm{P}<0.01)$. When comparing to the closed structure, significantly more inactive behavior was associated with both the neutral $(\mathrm{P}=0.01)$ and ramp structures $(\mathrm{P}<0.01)$. Vigilant behavior was more frequent at the ramp structure when compared to vigilant behavior at the closed $(\mathrm{P}<0.01)$ and neutral structures $(\mathrm{P}=0.05)$.

\section{DISCUSSION}

Captive coyotes spent a substantial amount of time at the perimeter and open areas of enclosures, but also overutilized shelter structures based on structure availability. Coyotes housed with complex enrichment structures also spent less time at the perimeter, an effect noticed in liontailed macaques (Mallapur et al., 2005). Novel and more complex enrichment structures were utilized more than the simple shade tables. Although there were three enrichment structures in the treatment enclosures and only two shade tables in the control enclosures, coyotes used enrichment structures more than twice as much as shade tables. This suggests the importance of providing additional complex enrichment structures for captive coyotes and illustrates the benefits of evaluating structural designs using different monitoring techniques. Models using the GPS and behavioral data produced similar estimates of enrichment structure preferences while providing unique predictive elements. Combining different monitoring techniques can help managers at captive animal facilities select biologically appropriate enrichment structure designs. Measuring the proportion of time at different structures is one method to spatially analyze the generic utility of a resource and decipher a preference for an area but must rely on direct or indirect methods to obtain the data. GPS-collars in this study described both nocturnal and diurnal patterns of captive coyotes. While it is beneficial to obtain information without human disturbance (Larsen, Sherwen, and Rault, 2014; Sekar, Rajagopal, and Archunan, 2008), GPS-collars only depict location and lack information on animal behavior. Behavioral assessments can help explain the functionality of resources in relation to the animal's inherent natural tendencies. Ethograms and activity budgets portraying behavioral repertoires of animals can be applied to illustrate animal responses to changes in their environment (Kluever and Gese, 2016; Wells and Hepper, 2000). For instance, these techniques have been useful in comparing the behavior of captive and wild coyote populations (Brummer et al., 2010; Shivik et al., 2009). Behavioral information collected from this study showed that complex enrichment structures were associated with predominantly inactive behavior, however, vigilant behavior occurred primarily at the ramp. Thus, using two discrete metrics improved estimates of the efficacy of environmental enrichment efforts and elucidated the biological and social functionality of different enclosure features.

Over-utilization and under-utilization of enclosure areas have been specifically measured to assess enclosure appropriateness and animal welfare of captive wild animals (Hunter et al., 2014; Ross et al., 2009). When evaluating the utilization of features in an animal's environment, only in theory will each resource be utilized proportionately to their allocated space. Animals naturally spend varying amounts of time exploiting different resources (Bekoff and Wells, 1981; Gese et al., 1996) and correlating these intricate biological functions with often crudely delineated spatial features is challenging. While over-utilized areas allude to associated features that are likely preferred, underutilized areas may suggest the avoidance of related resources and decrease the functional captive space. This study provides evidence that coyotes prefer shelter structures, especially those with more complex arrangements. Similar results have been found in studies of species that are prone to predation (Jensen, Gray, and Hurst, 2003; Kistler et al., 2010). Coyotes were mainly inactive at the enrichment structures, perhaps feeling more relaxed and secure in a more complex environment. Wild coyotes spend the majority of their time resting, especially in the winter months (Gese et al., 1996). Thus, any structure design that creates a more complex setting may be more amenable to a coyote's natural tendency to rest and display vigilance.

Although complex features in the enclosures were shown to be preferred, coyotes were still more frequently at the perimeter and open areas. Coyotes will routinely use howling and scent-marking for territory maintenance purposes and increase the frequency of scent-marking near territorial boundaries during the breeding season, December - February (Gese and Ruff, 1997). Perimeter areas of high intrusion are related to increased rates of raised-leg urinations (Wells and Bekoff, 1981). Similarly, captive coyotes often scent mark their enclosures and interact with neighbors while at the periphery (Schell, 
Young, Lonsdorf, Mateo, and Santymire, 2016). Coyotes in treatment enclosures spent less time at the perimeter than coyotes in control pens. A similar reduction in perimeter space use was observed in lion-tailed macaques when complex enrichment structures were introduced (Mallapur et al., 2005). Although perimeter utilization serves specific biological and social functions for captive coyotes, stereotypic pacing is often related to the peripheries of enclosures (Lyons, Young, and Deag, 1997), suggesting that structural features that reduce the time spent at the enclosure perimeter may improve well-being. Coyotes under-utilized the open areas of their enclosures. Open areas comprised more than half of the enclosure space and could analogously be considered as the core areas of their territories, which tend to remain stable over time (Young, Andelt, Terletzky, and Shivik, 2006). Aside from structural features, small prey such as voles and mice may naturally occur inside the enclosures and contribute to the utilization of areas. This may be why captive coyotes spend more time exploring their environment when housed in larger enclosures (Brummer et al., 2010). One would expect fewer small prey in the open, homogenous areas and more along the periphery, where they can escape predation by exiting the enclosure. Indeed, small mammals are often at higher abundance in edge habitats relative to homogenous landscapes (Bowers, Gregario, Brame, Matter, and Dooley, 1996). Wild coyotes generally avoid grasslands and prefer habitat that provide more structural complexity (i.e., pinyon-juniper and shrubs) which may be more abundant with prey (Gese, Rongstad, and Mytton, 1988). For captive coyotes, open areas are more homogenous than perimeter or structural features. Further, the preference for enrichment structures in treatment enclosures may have influenced this observed avoidance of open areas.

While a preference for one experimental enrichment structure did not materialize, some trends appeared. Behavioral scan observations, although only clustered during brief periods of daytime hours, showed that coyotes were more likely to be at the ramp structure than at the other two enrichment structures. This coincides with GPS-based modeled and observed results that male and female coyotes used the ramp structure more during the day rather than at night. GPS data also showed that when coyotes were located at an enrichment structure, they were most frequently recorded at the ramp. This may be explained by biological reasons; coyotes were mostly inactive at the enrichment structures, implying their suitability in offering protection from harsh environmental conditions common in winter. Vigilant behavior is routinely noticed in wild coyote populations, often in conjunction with resting (Bekoff and Wells, 1981). Similarly paired correlations were found in this study where vigilance was intermittently exhibited within longer lasting inactive states, and was most frequently recorded at the ramp structure. This suggests the ramp design may be best because it protects from weather and visual exposure, provides additional resting space, and better accommodates vigilant behavior with an accessible elevated platform.

The use of GPS-collars is a novel method for monitoring enclosure utilization of captive animals. While it provided a detailed evaluation of space use, even more detailed than noted in wild studies of coyotes (e.g., Arias-Del Razo, Hernández, Laundré, and Velasco-Vázquez, 2012), it also had limitations likely related to the GPS-collar design. The accuracy of the GPS-collars fluctuated in relation to their orientation to the sky, which would not have been known without the independent collar tests, because the GPSloggers did not record standard metrics of error (e.g., Bowman, Kochanny, Demarais, and Leopold, 2000; Frair et al., 2010; Hansen and Riggs, 2008). The GPS-collars were put on the coyotes in such a way that the data logger faced the sky when they were standing, sitting, or lying prone, and the collars successfully remained in that position on the neck for the duration of the testing period. Clusters of GPS points at the structure areas were readily discernable when visually inspecting the spatial distribution of the data, supporting that the collars were sensibly portraying animal spatial patterns. The high acquisition rates of the GPS-collars produced thousands of GPS points for each coyote, which helped validate the trends observed in this study. Using comparably large enclosures at the research facility (0.6ha enclosures rather than 0.1ha enclosures) helped account for GPS error by enabling the application of buffer areas. Enrichment structures are likely to be visible in future ESRI basemaps, making it feasible to reduce error even further in future studies.

While this study provides insight into enrichment structure preference by captive coyotes that may also be useful to other captive facilities, some limitations exist related to the methodology for obtaining behavioral information. While the use of a single observer eliminated any risk of inter-observer error, it introduced the 
possibility of intra-observer bias. Since behavior scores were recorded through live observations, intra-observer reliability unfortunately could not be examined. The observer used previously determined definitions to classify behavior and only three behavioral categories, which should reduce potential problems surrounding intra-observer error. However, with the consolidation of behavior to three states, specificity is lost and conflicting behavior can potentially occupy the same category. For example, active behavior would include aggressive behavior that may not be desirable for captive animals. In this study, less than one percent of active behavior included aggression and it does not appear that the new enrichment structures spurred undesirable behavior. Further, this categorical approach is commonly applied in research to gain statistical power (Busk and Marascuilo, 1992). Additionally, the observation window of 08:00 to 16:00 encompassed almost all of the daylight hours during the winter study period. It is likely that coyote activity levels fluctuated within this time span, potentially influencing results of this study. However, coyotes normally are inactive during this daytime period in winter months (Patterson et al., 1999; Way et al., 2004).

This study tested shelter structures that accommodated inactive behavior. It further evaluated their effectiveness by extracting vigilant behavior from the general inactive or active states. Similar methods have been applied to African wild dogs (Lycaon pictus), when the experiment categorized behavior into inactive, active, and social behavioral states (Rafacz and Santymire, 2013). Lastly, this study only compared between two experimental groups, and was not designed to monitor behavioral changes within groups. The experiment was conducted in this manner to reduce structure neophobia. Coyotes have been shown to be neophobic to new objects or stimuli (Harris and Knowlton, 2001; Mettler and Shivik, 2006). By installing the structures before coyote pairs were placed into the enclosure, a neophobic response was minimized but may not have been outright eliminated. Thus, having previous baseline behavioral assessment on treatment pairs could have improved our understanding of how the enrichment structures directly affected coyote behavior.

\section{CONCLUSIONS}

The provision of more complex enrichment structures increased coyote utilization of structures and reduced time spent at the enclosure perimeter. Coyotes overutilized all structure designs, and enclosures with more complex enrichment structures realized an under- utilization of open areas. Since no clear enrichment structure preference was evident, all three tested designs may be considered appropriate for coyotes in captivity. However, if only one design is applied, the ramp may be best because of the observed trend in greater use seen from both monitoring techniques. Further, the ramp provides easier access to the taller platform, offering additional versatility and utility for captive coyotes.

\section{ACKNOWLEDGMENTS}

We thank S. Brummer, M. Davis, N. Floyd, B. Jolley, E. Stevenson, E. Wright, and several volunteers with assistance conducting the field work. S. Friedman, E. Gese, and two anonymous reviewers provided excellent reviews of the manuscript. We also thank S. Durham for statistical advice. Funding was provided by USDANational Wildlife Research Center. Any use of trade, firm, or product names is for descriptive purposes only and does not imply endorsement by the U.S. Government.

\section{REFERENCES}

Altmann, J. 1974. Observational Study of Behavior: Sampling Methods. Behaviour, 49: 227-266.

Arias-Del Razo, I., L. Hernández, J. W. Laundré and L. VelascoVázquez. 2012. The landscape of fear: habitat use by a predator (Canis latrans) and its main prey (Lepus californicus and Sylvilagus audubonii). Canadian Journal of Zoology, 90: 683-693.

Arjo, W. M. and D. H. Pletscher. 1999. Behavioral responses of coyotes to wolf recolonization in northwestern Montana. Canadian Journal of Zoology, 77: 1919-1927.

Bates, D., M. Mächler, B. Bolker and S. Walker. 2015. Fitting Linear Mixed-Effects Models Usinglme4. Journal of Statistical Software, 67.

Begg, C. B. and R. Gray. 1984. Calculation of Polychotomous Logistic Regression Parameters Using Individualized Regressions. Biometrika, 71: 11.

Bekoff, M. and M. C. Wells. 1981. Behavioural budgeting by wild coyotes: The influence of food resources and social organization. Animal Behaviour, 29: 794-801.

Beyer, H. L., D. T. Haydon, J. M. Morales, J. L. Frair, M. Hebblewhite, M. Mitchell and J. Matthiopoulos. 2010. The interpretation of habitat preference metrics under use-availability designs. Philosophical Transactions of the Royal Society B: Biological Sciences, 365: 2245-2254.

Bloomsmith, M. A., L. Y. Brent and S. J. Schapiro. 1991. Guidelines for developing and managing an environmental enrichment program for nonhuman 
Coyote space use and structure preference

primates. Laboratory Animal Science, 41: 372-377.

Bowers, M. A., K. Gregario, C. J. Brame, S. F. Matter and J. L. Dooley. 1996. Use of space and habitats by meadow voles at the home range, patch and landscape scales. Oecologia, 105: 107-115.

Bowman, J. L., C. O. Kochanny, S. Demarais and B. D. Leopold. 2000. Evaluation of a GPS collar for whitetailed deer. Wildlife Society Bulletin: 141-145.

Brummer, S. P., E. M. Gese and J. A. Shivik. 2010. The effect of enclosure type on the behavior and heart rate of captive coyotes. Applied Animal Behaviour Science, 125: 171-180.

Busk, P. L. and L. A. Marascuilo. 2015. Statistical analysis in single-case research. Single-Case Research Design and Analysis (Psychology Revivals): New Directions for Psychology and Education: 159.

Frair, J. L., J. Fieberg, M. Hebblewhite, F. Cagnacci, N. J. DeCesare and L. Pedrotti. 2010. Resolving issues of imprecise and habitat-biased locations in ecological analyses using GPS telemetry data. Philosophical Transactions of the Royal Society B: Biological Sciences, 365: 2187-2200.

Gese, E. M., O. J. Rongstad and W. R. Mytton. 1988. Home Range and Habitat Use of Coyotes in Southeastern Colorado. The Journal of Wildlife Management, 52: 640.

Gese, E. M. and R. L. Ruff. 1997. Scent-marking by coyotes, Canis latrans: the influence of social and ecological factors. Animal Behaviour, 54: 1155-1166.

Gese, E. M., R. L. Ruff and R. L. Crabtree. 1996. Foraging ecology of coyotes (Canis latrans): the influence of extrinsic factors and a dominance hierarchy. Canadian Journal of Zoology, 74: 769-783.

Gilbert-Norton, L. B., L. A. Leaver and J. A. Shivik. 2009. The effect of randomly altering the time and location of feeding on the behaviour of captive coyotes (Canis latrans). Applied Animal Behaviour Science, 120: 179-185.

Gilbert-Norton, L. B., R. R. Wilson and J. A. Shivik. 2013. The Effect of Social Hierarchy on Captive Coyote (Canis latrans) Foraging Behavior. Ethology, 119: 335-343.

Grow, S., R. Allard and D. Luke. 2015. The Role of AZAAccredited Zoos and Aquariums in Butterfly Conservation. Butterfly Conservation in North America. Springer Netherlands, pp. 23-34.

Hansen, M. C. and R. A. Riggs. 2008. Accuracy, Precision, and Observation Rates of Global Positioning System Telemetry Collars. Journal of Wildlife Management, 72: 518-526.

Harris, C. E. and F. F. Knowlton. 2001. Differential responses of coyotes to novel stimuli in familiar and unfamiliar settings. Canadian Journal of Zoology, 79: 2005-2013.

Hunter, S. C., M. Gusset, L. J. Miller and M. J. Somers. 2014. Space Use as an Indicator of Enclosure Appropriateness in African Wild Dogs (Lycaon pictus). Journal of Applied Animal Welfare Science, 17: 98-110.

Jensen, S. P., S. J. Gray and J. L. Hurst. 2003. How does habitat structure affect activity and use of space among house mice? Animal Behaviour, 66: 239250.

Kistler, C., D. Hegglin, H. Würbel and B. König. 2010. Structural enrichment and enclosure use in an opportunistic carnivore: the red fox (Vulpes vulpes). Animal Welfare, 19: 391.

Kluever, B. M. and E. M. Gese. 2016. Spatial response of coyotes to removal of water availability at anthropogenic water sites. Journal of Arid Environments, 130: 68-75.

Kuczaj, S., T. Lacinak, O. Fad, M. Trone, M. Solangi and J. Ramos. 2002. Keeping environmental enrichment enriching. International Journal of Comparative Psychology, 15.

Larsen, M. J., S. L. Sherwen and J.-L. Rault. 2014. Number of nearby visitors and noise level affect vigilance in captive koalas. Applied Animal Behaviour Science, 154: 76-82.

Law, G. and A. Reid. 2010. Enriching the lives of bears in zoos. International zoo yearbook, 44: 65-74.

Lenth, R. V. 2016. Least-Squares Means: TheRPackagelsmeans. Journal of Statistical Software, 69.

Lyons, J., R. J. Young and J. M. Deag. 1997. The effects of physical characteristics of the environment and feeding regime on the behavior of captive felids. Zoo Biology, 16: 71-83.

Mallapur, A., Q. Qureshi and R. Chellam. 2002. Enclosure Design and Space Utilization by Indian Leopards (Panthera pardus) in Four Zoos in Southern India. Journal of Applied Animal Welfare Science, 5: 111-124.

Mallapur, A., N. Waran and A. Sinha. 2005. Use of Enclosure Space by Captive Lion-Tailed Macaques (Macaca silenus) Housed in Indian Zoos. Journal of Applied Animal Welfare Science, 8: 175-186.

Mellen, J. and M. Sevenich MacPhee. 2001. Philosophy of environmental enrichment: Past, present, and future. Zoo Biology, 20: 211-226.

Mettler, A. E. and J. A. Shivik. 2007. Dominance and neophobia in coyote (Canis latrans) breeding pairs. Applied Animal Behaviour Science, 102: 85-94. 
Mills, L. S. and F. F. Knowlton. 1991. Coyote space use in relation to prey abundance. Canadian Journal of Zoology, 69: 1516-1521.

Newberry, R. C. 1995. Environmental enrichment: Increasing the biological relevance of captive environments. Applied Animal Behaviour Science, 44: 229-243.

Patterson, B., S. Bondrup-Nielsen and F. Messier. 1999. Activity patterns and daily movements of the eastern coyote, Canis latrans, in Nova Scotia. Canadian Field-Naturalist, 113: 251-257.

Rafacz, M. L. and R. M. Santymire. 2013. Using odor cues to elicit a behavioral and hormonal response in zoo-housed African wild dogs. Zoo Biology, 33: 144-149.

Renner, M. J. and J. P. Lussier. 2002. Environmental enrichment for the captive spectacled bear (Tremarctos ornatus). Pharmacology Biochemistry and Behavior, 73: 279-283.

Ross, S. R., S. J. Schapiro, J. Hau and K. E. Lukas. 2009. Space use as an indicator of enclosure appropriateness: A novel measure of captive animal welfare. Applied Animal Behaviour Science, 121: 42-50.

Samuelson, M. M., L. K. Lauderdale, K. Pulis, M. Solangi, T. Hoffland and H. Lyn. 2016. Olfactory Enrichment in California Sea Lions (Zalophus californianus): An Effective Tool for Captive Welfare? Journal of Applied Animal Welfare Science, 20: 75-85.

Schell, C. J., J. K. Young, E. V. Lonsdorf, J. M. Mateo and R. M. Santymire. 2016. Olfactory attractants and parity affect prenatal androgens and territoriality of coyote breeding pairs. Physiology and Behavior, 165: 43-54.

Sekar, M., T. Rajagopal and G. Archunan. 2008. Influence of Zoo Visitor Presence on the Behavior of Captive Indian Gaur (Bos gaurus gaurus) in a Zoological
Park. Journal of Applied Animal Welfare Science, 11: 352-357.

Shivik, J. A., M. M. Jaeger and R. H. Barrett. 1996. Coyote Movements in Relation to the Spatial Distribution of Sheep. The Journal of Wildlife Management, 60: 422.

Shivik, J. A., G. L. Palmer, E. M. Gese and B. Osthaus. 2009. Captive Coyotes Compared to Their Counterparts in the Wild: Does Environmental Enrichment Help? Journal of Applied Animal Welfare Science, 12: 223-235.

Skaug, H., D. Fournier, A. Nielsen, A. Magnusson and B. Bolker. 2013. Generalized linear mixed models using AD model builder. R package version 0.7, 7.

Team, R. C. 2016. R: A language and environment for statistical computing. R Foundation for Statistical Computing, Vienna, Austria. 2014.

Way, J. G., I. M. Ortega and E. G. Strauss. 2004. Movement and Activity Patterns of Eastern Coyotes In a Coastal, Suburban Environment. Northeastern Naturalist, 11: 237-254.

Wells, D. L. 2009. Sensory stimulation as environmental enrichment for captive animals: A review. Applied Animal Behaviour Science, 118: 1-11.

Wells, D. L. and P. G. Hepper. 2000. The influence of environmental change on the behaviour of sheltered dogs. Applied Animal Behaviour Science, 68: 151-162.

Wells, M. C. and M. Bekoff. 1981. An observational study of scent-marking in coyotes, Canis latrans. Animal Behaviour, 29: 332-350.

Young, J. K., W. F. Andelt, P. A. Terletzky and J. A. Shivik. 2006. A comparison of coyote ecology after 25 years: 1978 versus 2003 This is Welder Wildlife Foundation Contribution No. 651. Canadian Journal of Zoology, 84: 573-582.

Publisher's note: EScience Press remains neutral with regard to jurisdictional claims in published maps and institutional affiliations.

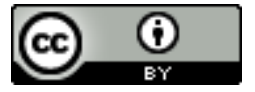

Open Access This article is licensed under a Creative Commons Attribution 4.0 International License, which permits use, sharing, adaptation, distribution and reproduction in any medium or format, as long as you give appropriate credit to the original author(s) and the source, provide a link to the Creative Commons license and indicate if changes were made. The images or other third-party material in this article are included in the article's Creative Commons license, unless indicated otherwise in a credit line to the material. If material is not included in the article's Creative Commons license and your intended use is not permitted by statutory regulation or exceeds the permitted use, you will need to obtain permission directly from the copyright holder. To view a copy of this license, visit http://creativecommons.org/licenses/by/4.0/.

(C) The Author(s) 2019. 